\title{
Investigation of neurotrophic tyrosine kinase receptor 1 fusions and neurotrophic tyrosine kinase receptor family expression in non-small-cell lung cancer and sensitivity to AZD7451 in vitro
}

\author{
TSUTOMU TATEMATSU ${ }^{1}$, HIDEFUMI SASAKI ${ }^{1}$, SHIGEKI SHIMIZU ${ }^{2}$, KATSUHIRO OKUDA $^{1}$, \\ MASAYUKI SHITARA $^{1}$, YU HIKOSAKA ${ }^{1}$, SATORU MORIYAMA ${ }^{1}$, MOTOKI YANO ${ }^{1}$, \\ JEFFREY BROWN $^{3}$ and YOSHITAKA FUJII ${ }^{1}$
}

\footnotetext{
${ }^{1}$ Department of Oncology, Immunology and Surgery, Nagoya City University Graduate School of Medical Sciences, Nagoya, Aichi 467-8601; ${ }^{2}$ Department of Pathology, Hyogo College of Medicine, Nishinomiya, Hyogo 663-8501, Japan;

${ }^{3}$ AstraZeneca Pharmaceuticals, Waltham, MA 20451, USA
}

Received April 14, 2014; Accepted May 29, 2014

DOI: $10.3892 / \mathrm{mco} .2014 .318$

\begin{abstract}
Advances in the molecular segmentation of lung cancer has raised the possibility that neurotrophic tyrosine kinase receptor (NTRK) 1 fusions and NTRK1-3 expression may be promising molecular targets for future therapeutic interventions. We investigated the antitumor effects of a selective pan-NTRK inhibitor, AZD7451, by evaluating its antiproliferative effects on the KM12 cell line (a colorectal cancer cell line harboring a tropomyosin-NTRK1 fusion) and the $\mathrm{H} 460$ and $\mathrm{H} 810$ cell lines [large-cell neuroendocrine carcinoma (LCNEC) cell lines expressing NTRK2]. Relative quantitative polymerase chain reaction (qPCR) was performed to measure the mRNA levels of the NTRK1-3 tyrosine kinase domain using cDNA extracted from KM12, H460 and H810 cells. The cultures were grown in 6-well plates at a density of $1.0 \times 10^{6}$ cell/well and treated with AZD7451 at different doses $(1,2.5,4,5,7.5$ and $10 \mathrm{nM})$ using dimethyl sulfoxide as a control. Following a 24-h incubation, the number of surviving cells was measured using a hemocytometer. Furthermore, we performed western blotting of the high-affinity nerve growth factor receptor (TRKA) and NTRK2 (TRKB) proteins and monitored the effects on the downstream signaling pathways Akt and ERK in these cell lines following treatment with AZD7451 (KM12 and H460: 0, 1 and $5 \mathrm{nM}$; H810: 0 and $5 \mathrm{nM}$ ). Immunohistochemical analyses of the surgically resected samples were also performed, using anti-NTRK1,2 antibodies. We performed reverse-transcription PCR and direct sequencing to investigate NTRK fusions in 268 patients;
\end{abstract}

Correspondence to: Dr Hidefumi Sasaki, Department of Oncology, Immunology and Surgery, Nagoya Graduate School of Medical Sciences, Nagoya City University, 1 Kawasumi, Mizuho-cho, Mizuho-ku, Nagoya, Aichi 467-8601, Japan

E-mail: hisasaki@hotmail.com

Key words: neurotrophic tyrosine kinase receptor, AZD7451, fusion, KM12 however, were unable to confirm the presence of NTRK fusions in this cohort. Further immunohistochemical analyses of the primary patient samples demonstrated that none of 61 tumors had NTRK1 overexpression and 7 of 39 samples exhibited NTRK2 expression, including 1 LCNEC sample. The qPCR results from the KM12 cell line revealed an apparent increase and overexpression of NTRK1 mRNA levels, while H460 cells exhibited a modest increase and the H810 cell line showed no apparent increase in the expression of any NTRK1-3 isoforms. There were no increases in the NTRK 2 mRNA levels in any of the three cell lines, although KM12 and H460 cells exhibited low levels of NTRK2 expression. In vitro growth and proliferation of the KM12 cell line harboring the NTRK1-fusion was found to be potently inhibited by AZD7451 at a concentration of $2 \mathrm{nM}$. The proliferation of $\mathrm{H} 460$ cells was also found to be inhibited at a concentration of $5 \mathrm{nM}$, while there was no apparent inhibitory effect of AZD7451 on the growth or proliferation of $\mathrm{H} 810$ cells. Western blotting of KM12 cells treated with AZD7451 also revealed a potent inhibition of TRKA phosphorylation following AZD7451 treatment. Analysis of $\mathrm{H} 460$ cells confirmed the expression and inhibition of phosphorylation of NTRK2, whereas there was little to no expression of TRKA/B in H810 cells. Subsequent in vitro analysis of cell lines treated with the pan-TRK inhibitor AZD7451 suggested that the proliferation of KM12 and $\mathrm{H} 460$ cells was significantly inhibited by AZD7451, while H810 cells expressing low levels of wild-type NTRK1-3 were not inhibited. Based on these results, there is potential for a NTRK-dependent proliferation driver in a subpopulation of lung cancer patients with NTRK expression. In addition, pharmacological inhibition with a NTRK inhibitor, such as AZD7451, in cells harboring NTRK1 fusions, may be associated with beneficial antitumor effects.

\section{Introduction}

Lung cancer, which is usually diagnosed at an advanced stage, is a major cause of cancer-related mortality, due to its high incidence, aggressive behavior and lack of major advancements 
in treatment strategy (1). Lung cancer was the leading indication for respiratory surgery (48.9\%) in 2011 in Japan (2) and $>33,000$ patients underwent surgery for lung cancer at Japanese institutions in the same year (2). The clinical behavior of non-small-cell lung cancer (NSCLC) is largely associated with cancer stage. Curative surgery is only achieved in early-stage NSCLC (3). Recent advances in targeted therapy for NSCLC have augmented treatment choices for lung adenocarcinoma, but chemotherapy remains the therapeutic mainstay for other types of NSCLC.

Molecular-targeted therapies, such as crizotinib, which target anaplastic lymphoma kinase fusion gene, and erlotinib or gefitinib, which target epidermal growth factor receptor gene mutations, have demonstrated superior single-agent activity in selected patients compared to standard chemotherapy regimes in lung cancer treatment $(4,5)$. Recently, a series of new gene fusions were described in lung cancer patients associated with the kinase domain of the NTRK1 gene, which encodes the high-affinity nerve growth factor receptor (TRKA) protein (6). Both the myosin phosphatase Rho-interacting protein (MPRIP)-NTRK1 and CD74-NTRK1 fusions lead to constitutive TRKA kinase activity and have been shown to be oncogenic. In addition, Marchetti et al (7) demonstrated that there are NTRK3 mutations associated with the TRKC receptor and NTRK2 mutations encoding the NTRK2 (TRKB) receptor in lung large-cell neuroendocrine carcinoma (LCNEC). Recently, the TRKB signaling pathway was also reported to be a potential therapeutic target for lung LCNEC (8).

NTRK1 fusions and NTRK expression in lung cancer may be promising as a molecular-targeted therapy for future clinical trials. To expand these findings and determine the prevalence of these mutations in a cohort of Japanese lung cancer patients, we investigated the presence of NTRK1 fusions in surgical resection NSCLC samples (adenocarcinoma, 198 cases; and squamous cell carcinoma, 70 cases). Surprisingly, using reverse-transcription polymerase chain reaction (RT-PCR) and direct DNA sequencing, we were unable to identify fusions in any of those patients. The immunohistochemical analysis demonstrated that some lung cancer cases exhibited NTRK2 expression. For this reason, we further investigated the in vitro antitumor effects of AZD7451 on the KM12 cell line [colorectal cancer cell line harboring tropomyosin (TPM)-NTRK1 fusion] (6) and the H460 and H810 cell lines (LCNEC cell lines exhibiting NTRK2 expression). AZD7451 is a potent small-molecule pan-TRK inhibitor with a high degree of specificity and selectivity as compared to other kinases (9). We also performed a PCR using total mRNA extracted from the three cell lines and examined the total mRNA expression levels in these cell lines.

\section{Patients and methods}

PCR assays for NTRK1 fusions. Total RNA was extracted from lung cancer tissues and adjacent normal lung tissues using Isogen kit (Nippon Gene, Tokyo, Japan) according to the manufacturer's instructions. RNA concentration was determined using a spectrophotometer (NanoDrop Technologies, Inc., Rockland, DE, USA) and adjusted to a concentration of $200 \mathrm{ng} / \mathrm{ml}$. RNA (1 $\mu \mathrm{g})$ was reverse-transcribed using
Superscript II enzyme (Gibco-BRL, Gaithersburg, MD, USA) with $0.5 \mu \mathrm{g}$ oligo(dT) ${ }_{12-16}$ (Amersham Pharmacia Biotech, Inc., Piscataway, NJ, USA). DNA concentration was determined by a NanoDrop spectrophotometer and adjusted at $50 \mathrm{ng} / \mathrm{ml}$. We used $1 \mathrm{ml}$ of each DNA for the assays. The fusion gene primers were 5'-CTCCCAAGCCTGTGAGCAAGAT-3' (CD74; forward), 5'-AGGAGATTAGCTCCCTCAAGGAT-3' (MPRIP; forward) and 5'-GTTGTGGCACTCAGCAAG GAAG-3' (NTRK1; reverse) and they were amplified (6). The cycling conditions were as follows: initial denaturation at $94^{\circ} \mathrm{C}$ for $1 \mathrm{~min}$, followed by 40 cycles at $98^{\circ} \mathrm{C}$ for $10 \mathrm{sec}$ and $68^{\circ} \mathrm{C}$ for $60 \mathrm{sec}$, followed by a final extension at $72^{\circ} \mathrm{C}$ for $10 \mathrm{~min}$.

Cell lines and cell culture. The KM12 colorectal cancer cell line and the H460 and H810 LCNEC cell lines were purchased from American Type Culture Collection (Manassas, VA, USA). The KM12 and H460 cell lines were maintained in RPMI-1640 medium with 10\% fetal bovine serum (FBS). H810 cells were maintained in HITES medium supplemented with $5 \% \mathrm{FBS}$. All the cell lines were grown at $37^{\circ} \mathrm{C}$ in a humidified incubator with $5 \% \mathrm{CO}_{2}$. The cells were grown to $60-70 \%$ confluence, harvested with trypsin and resuspended to the cell density required for each assay.

qPCR of NTRK1-3. Total RNA was prepared from the KM12, H460 and H810 cell lines using the miRNeasy mini kit (Qiagen, Hilden, Germany) and was reverse-transcribed using the High-Capacity cDNA Reverse Transcription kit (Applied Biosystems, Foster City, CA, USA) according to the manufacturer's instructions. Relative qPCR of the NTRK1-3 tyrosine kinase domains (NTRK1, Hs01021011_m1; NTRK2, Hs00178811_m1; and NTRK3, 00176797_m1; Applied Biosystems) was used to evaluate their level of mRNA expression. The relative quantification method in the 7500 Fast Real-time PCR system (Applied Biosystems) was used, with actin- $\beta$ (Applied Biosystems) as an endogenous internal control. All the samples were evaluated in triplicate. Approximately 80 lung cancer cases were also evaluated for NTRK1-3 expression.

Immunohistochemistry. NTRK protein expression was evaluated by immunohistochemistry using rabbit monoclonal anti-TRKA (Sab76291; Abcam, Cambridge, MA, USA) and anti-TRKB (80G2; Cell Signaling Technology, Boston, MA, USA) antibodies. We used a standard protocol for the immunostaining of samples as previously described (8). Sections $(4 \mu \mathrm{m})$ were cut from paraffin tissue blocks from NSCLC tumors and mounted onto glass slides. The slides were treated with xylene and then dehydrated in graded alcohols. For epitope retrieval, the specimens were exposed to $10 \mathrm{mM}$ citrate buffer ( $\mathrm{pH}$ 6.0) and heated to $\sim 10 \mathrm{~min}$ in autoclave. Endogenous peroxidase activity was blocked with $\mathrm{H}_{2} \mathrm{O}_{2}$ in methanol. The sections were incubated with blocking solution (10\% Block Ace) and then allowed to react with anti-TRKA (x100) or anti-TRKB (x80) antibody overnight at $4^{\circ} \mathrm{C}$. After the excess antibody had been washed out with phosphate-buffered saline (PBS), the samples were incubated with a peroxidase-conjugated anti-rabbit antibody (rabbit HRP EnVision ${ }^{\mathrm{TM}+}$; Dako, Carpinteria, CA, USA) for $45 \mathrm{~min}$. After the excess antibody had been washed out with PBS, 3,3-diaminobenzidine substrate (10 min) was used 



Figure 1. A relative quantitative polymerase chain reaction assay was performed to measure the mRNA levels of the NTRK1-3 tyrosine kinase domain in the three cell lines (left) and tumor and normal tissues from large-cell neuroendocrine carcinoma (LCNEC) (right). (A) The KM12 cell line exhibited high NTRK1 mRNA levels, whereas $\mathrm{H} 460$ cells exhibited intermediate expression. (B and C) There was no increase in the mRNA levels for NTRK2 and NTRK3 in any of the cell lines. (A-C) The H810 cell line showed relatively low NTRK1-3 mRNA levels. (D) Four primary patient samples of LCNEC were resected at our institution; however, we did not observe evidence of increased mRNA levels in the tumor cells compared to those in normal tissue. t1-4, tumor tissues from LCNEC samples; n1-4, normal tissues from the LCNEC samples. NTRK, neurotrophic tyrosine kinase receptor.

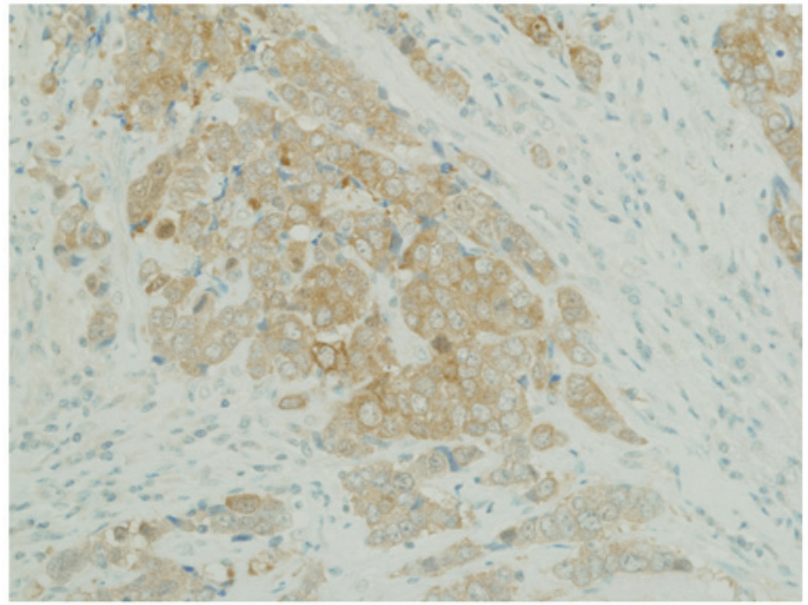

Figure 2. TRKB immunohistochemistry result. Large-cell neuroendocrine carcinoma expressing NTRK2. NTRK, neurotrophic tyrosine kinase receptor.

to visualize antibody binding and the sections were counterstained with hematoxylin. NTRK staining was evaluated under a light microscope (Leica DM4000B; Leica microsystems, Wetzler, Germany) with a magnification of $x 400$.

Evaluation of growth inhibition by AZD7451 in vitro. To determine whether NTRK fusions or mutations were capable of driving cell growth and proliferation in vitro, we used AZD7451 (AstraZeneca Pharmaceuticals, Waltham, MA, USA) to inhibit NTRK isoforms in the three cell lines. AZD7451 is a potent small-molecule pan-TRK inhibitor, which has been reported to have a similar $2-5 \mathrm{nM}$ in vitro enzyme potency in cell-based assays (5).

KM12, H460 and H810 cells were plated in 6-well plates at density of $1.0 \times 10^{6}$ cell/well at $24 \mathrm{~h}$ following treatment with AZD7451 at variable doses (1, 2.5, 4, 5, 7.5 and $10 \mathrm{nM})$. Dimethyl sulfoxide (DMSO) was used as a negative control. After $24 \mathrm{~h}$, the cells were counted using a hemocytometer and the data are expressed as percent control (\% control), which represents the ratio of cells treated with AZD7451 against control cells (Fig. 3).
Western blotting and antibodies. Protein extracts from KM12, H460 and H810 cells were used for western blotting to measure the relative expression of NTRK protein levels. The cells were cultured at density of $1.0 \times 10^{6}$ cell/well in 6 -well plates as previously described. KM12 and H460 cells were used after being treated with AZD7451 concentrations of 0,1 and $5 \mathrm{nM}$ and incubated for $24 \mathrm{~h}$. H810 cells were also used after being treated with a concentration of $0,1 \mathrm{nM}$ as control.

After treatment, the cultured cells were washed, scraped and resuspended in NuPAGE lithium dodecyl sulfate sample buffer (Invitrogen Life Technologies, Carlsbad, CA, USA). Equal amounts of protein were separated by SDS-PAGE and electroblotted onto polyvinylidene difluoride membranes (Bio-Rad, Hercules, CA, USA). The membranes were blocked in PBS containing $0.1 \%$ Tween-20 and 5\% ECL blocking agent (GE Healthcare, Little Chalfont, UK) and probed with primary antibodies directed against pAkt (Ser473, 1:5,000), pERK1/2 (Thr202/Tyr204, 1:5,000), pTRKA/B (Tyr490/Tyr512, 1:1,000),pTRKA/B (Tyr674/675/Tyr706/707, 1:1,000) and total TRKB $(1: 2,000)$ (Cell Signaling Technology), total TRKA $(1: 1,000)$ and $\alpha$-tubulin $(1: 5,000)$ (Santa Cruz Biotechnology, Inc., Santa Cruz, CA, USA). The membranes were washed and incubated with secondary antibody consisting of horseradish peroxidase-linked anti-rabbit or anti-mouse $\mathrm{IgG}$ (GE Healthcare), at a dilution of 1:5,000.

\section{Results}

Absence of NTRK1 fusion genes in Japanese patients. According to Vaishnavi et al (6), we investigated whether CD74-NTRK1 or MPRIP-NTRK1 fusions existed in a cohort of 268 primary samples resected from Japanese NSCLC patients. Within 198 adenocarcinomas, 70 were known driver mutation-negative cases. Using RT-PCR and direct DNA sequencing, we were unable to identify fusions in any of these cases.

Total mRNA levels of NTRK1-3 tyrosine kinase domain. Relative qPCR assay was performed to measure the mRNA levels of the NTRK1-3 tyrosine kinase domain using cDNA 

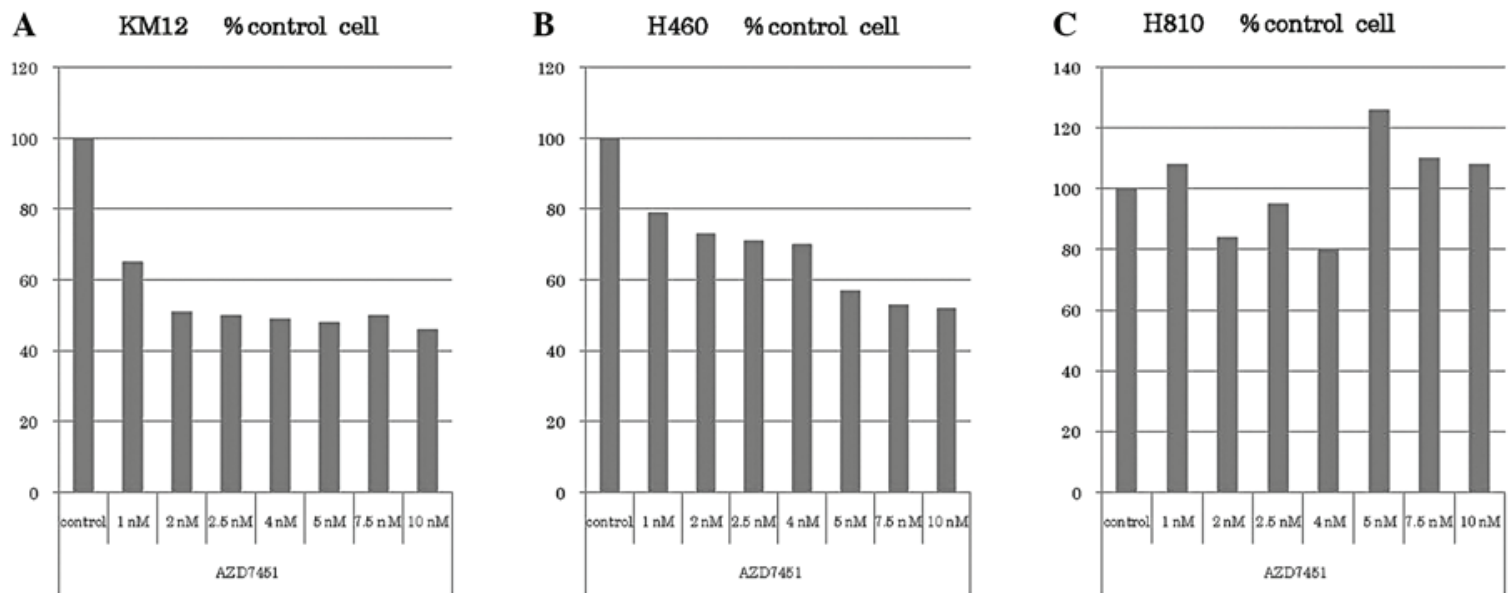

Figure 3. KM12, $\mathrm{H} 460$ and $\mathrm{H} 810$ cells were plated in 6-well plates at a density of 1.0x106 cells/well at $24 \mathrm{~h}$ after being treated with AZD7451 at variable doses $(1,2.5,4,5,7.5$ and $10 \mathrm{nM})$. DMSO was used as a negative control. After $24 \mathrm{~h}$, the cells were counted using a hemocytometer. (A) KM12 cell proliferation was potently inhibited at a concentration of $1 \mathrm{nM}$. Further cell proliferation was inhibited up to $2 \mathrm{nM}$, corresponding to a growth suppression of $\sim 60 \%$. (B) $\mathrm{H} 460$ cell proliferation was also inhibited at concentrations $\geq 1 \mathrm{nM}$. Further cell proliferation was inhibited up to $5 \mathrm{nM}$, also with a maximal suppression of growth of $\sim 60 \%$. (C) There was no apparent inhibitory effect on H810 cell proliferation. \%control, ratio of cells treated with AZD7451 against control cells.

extracted from three cell lines, namely KM12, H460 and H810. Our results demonstrated that the KM12 cell line exhibited high levels of NTRK1 mRNA, the H460 cell line exhibited intermediate expression, while the H810 cell line exhibited relatively low NTRK1-3 mRNA levels. There was no increase in the NTRK2 mRNA levels in any of the three cell lines, although KM12 and H460 cells exhibited a marginal increase in NTKR2 expression (Fig. 1). In addition, relative qPCR was also performed on 4 primary samples of LCNEC resected at our institution, but we found no evidence of increased NTRK mRNA levels in tumor cells compared to those in normal tissue. In adenocarcinomas, 4/74 exhibited high NTRK1 expression (tumor/normal ratio, >1), 3/66 exhibited high NTRK2 expression and 1/62 exhibited high NTRK3 expression.

Immunohistochemical analyses for NTRK. Using the immunohistochemical analysis described above for NTRK1, we investigated NTRK1 protein expression in 61 lung cancer samples; however, none of the samples exhibited significant levels of detectable NTRK1 (TRKA) overexpression. In addition, we investigated NTRK2 (TRKB) expression and found that 7 of the 61 patient samples stained positive for NTRK2. In addition, 1 of the 4 LCNEC samples stained strongly positive for NTKR2 (Fig. 2).

Cytostatic effect of AZD7451 in vitro. The KM12, H460 and H810 cell lines were treated with various doses of AZD7451 $(0,1,2.5,4.5,7.5$ and $10 \mathrm{nM})$ and the results are presented in Fig. 3. KM12 cell proliferation was potently inhibited at a concentration of $1 \mathrm{nM}$, as compared to the DMSO control. Further cell proliferation was inhibited up to $2 \mathrm{nM}$, corresponding to a growth suppression of $\sim 60 \%$; however, there were no further differences in cell proliferation with increased concentrations of AZD7451. H460 cell proliferation was also inhibited at concentrations $\geq 1 \mathrm{nM}$. Further cell proliferation was inhibited up to $5 \mathrm{nM}$, also with a maximal suppression of growth at $\sim 60 \%$. There was no apparent inhibitory effect on H810 cell proliferation.

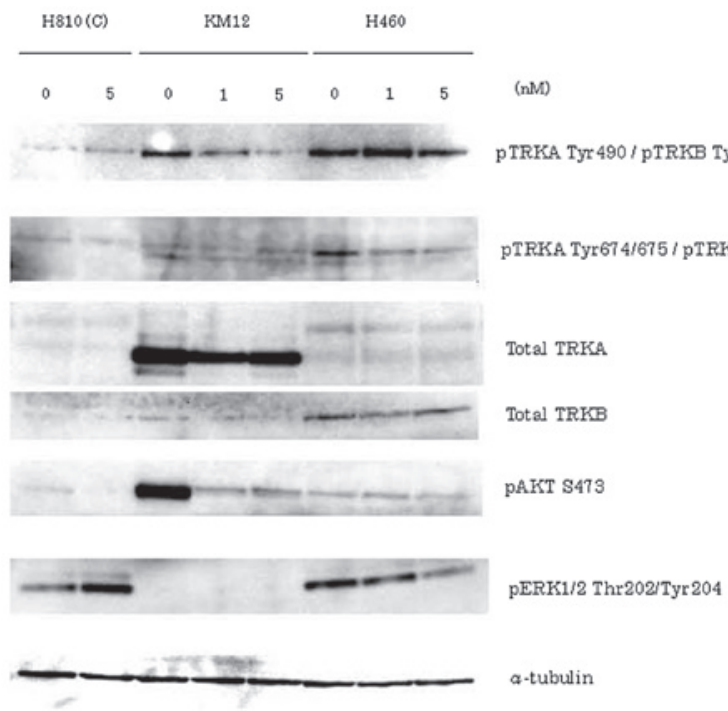

Figure 4. In order to determine TRKA/B phosphorylation and the downstream signaling effects on Akt and ERK, western blotting was performed in KM12, H460 and H810 cells. KM12 and H460 cells were treated with AZD7451 at concentrations of 0,1 and $5 \mathrm{nM}$, and incubated for $24 \mathrm{~h}$. H810 cells were also used as control, after being treated at concentrations of 0 and $5 \mathrm{nM}(\mathrm{C})$. In KM12 cells, the potent inhibition of pTRKA Tyr 490 and pAkt was found to be concentration-dependent; however, the inhibition of the expression of pTRKA Tyr674/675 and pERK was unclear. In H460 cells, total TRKB was strongly expressed compared to total TRKA. In addition, the expression of pTRKB Tyr706/707 and pERK was inhibited in a drug concentration-dependent manner, but inhibition of the expression of pTRKB Tyr512 and pAkt was unclear in H460 cells. H810 cells exhibited little to no expression of TRKA/B.

Inhibition of phosphorylation of TRKA/B and downstream signaling in cells treated with AZD7451. In order to determine the phosphorylation of TRKA/B and the downstream signaling effects on Akt and ERK, we performed western blotting in all three cell lines treated with AZD7451 at different doses (KM12 and H460: 0, 1 and $5 \mathrm{nM}$; and H810: 0 and $5 \mathrm{nM}$ ) (Fig. 4). In KM12 cells, the potent inhibition of pTRKA Tyr490 and pAkt was found to be concentration-dependent, 
but inhibition of the expression of pTRKA Tyr674/675 and pERK was unclear. In H460 cells, total TRKB was strongly expressed compared to the total TRKA. Therefore, we considered that H460 cells primarily express NTRK2. In addition, the expression of pTRKB Tyr706/707 and pERK was inhibited in a concentration-dependent manner, but inhibition of the expression of pTRKB Tyr512 and pAkt was unclear in H460 cells. H810 cells exhibited little to no expression of TRKA/B.

\section{Discussion}

Vaishnavi et al (6) previously reported the presence of NTRK1 fusions in 3 of 91 (3.3\%) NSCLC cases. In addition, Marchetti et al (7) also reported the presence of NTRK2,3 mutations (NTRK2 in 4 and NTRK3 in 5 cases) in 9 of 29 cases (31\%) of LCNEC. In this study, we sought to confirm and extend these analyses and determine the prevalence of these mutations in a specific cohort of 203 Japanese NSCLC patients. Understanding the specific molecular segments and proliferation drivers may improve lung cancer treatment in Japanese patients in the future. Therefore, we used RT-PCR, direct sequencing, immunostaining and qPCR in 268 cases of NSCLC specimens from our institution. Patients with specific mutations (data not shown) or translocations of the NTRK genes were not identified. There is a possibility that the frequency of the genetic abnormalities is extremely low in the Japanese patient population, or that there are biological differences in the disease subtypes used in this study. However, to determine the underlying causes of these differences, more patient samples and additional data associated with environmental factors are required to further validate and confirm these results in the future.

NTRK2 (TRKB) has been suggested to play a role in various neuroendocrine cancers; however, there is no direct evidence supporting a role for any of the NTRK1-3 receptors in cancer. The expression of NTKR2 and its ligand brain-derived neurotrophic factor (BDNF) analyzed by immunohistochemistry for lung cancer were found to be significantly higher in neuroendocrine tumors (NET) compared to non-NET (8). In particular, LCNEC, a subtype of NET, exhibited significantly higher NTRK2 and BDNF compared to another NET type, small-cell lung cancer; and a significant correlation between NTRK2 and BDNF expression was noted in LCNEC, but not in small-cell lung cancer (5). In an in vitro assay, the addition of exogenous BDNF enhanced the invasion of matrigel by LCNEC cells, whereas the inhibition of NTRK 2 or BDNF suppressed matrix metalloproteinase activity and tumor cell invasiveness (8). Exogenous BDNF also increased anchor-independent colony formation on soft agar gels for LCNEC, while the inhibition of NTRK2 or BDNF suppressed anchorage-independent growth. In vivo experiments using implanted LCNEC cells pretreated with NTRK2-siRNA failed to develop subcutaneous tumors, while most the control-treated siRNA cells were capable of forming tumors in nude mice $(8,10)$. LCNEC is associated with a particularly poor prognosis and an effective therapeutic strategy has yet to be established. Thus, BDNF/NTRK2 signaling appears to be involved in malignant progression, invasiveness and tumorigenicity of LCNEC and may be a potential target for LCNEC patients without other options for standard cancer therapy.
We further examined the mRNA levels for the tyrosine kinase domain of NTRK1-3 and the potential antitumor effects of AZD7451 in cell lines expressing TPM-NTRK1 fusion and those exhibiting NTRK2 overexpression. The KM12 and H460 cell lines exhibited increased expression of NTRK1 mRNA levels, while the H810 cell line did not exhibit significant NTRK1-3 mRNA levels. Interestingly, none of the three cell lines exhibited increased mRNA levels of NTRK2,3 based on RT-qPCR data (Fig. 1). Moreover, when the three cell lines were treated with AZD7451, the proliferation of H460 and KM12 cells was significantly inhibited at concentrations of 1-2 nM, but the proliferation of $\mathrm{H} 810$ cells was not inhibited at significantly higher doses (Fig. 2). Based on these results, we hypothesized that the expression of NTRK1 and a potential proliferation drive may both be associated with NTRK in the KM12 and H460 cell lines.

To further test this hypothesis, we examined the phosphorylation of TRKA/B, Akt and ERK1/2. Active signaling was confirmed by western blotting and subsequent inhibition in the three cell lines treated with AZD7451 at various doses (KM12 and H460: 0, 1 and $5 \mathrm{nM}$; and H810: 0 and $5 \mathrm{nM}$ ) (Fig. 4). In KM12, the expression of pTRKA Tyr490 and pAkt was potently inhibited in a drug concentration-dependent manner, but inhibition of the expression of pTRKA Tyr674/675 and pERK was unclear. In H460 cells, total TRKB was strongly expressed compared to total TRKA. Therefore, we considered that $\mathrm{H} 460$ cells predominantly express NTRK2. In addition, the expression of pTRKB Tyr706/707 and pERK was inhibited in a drug concentration-dependent manner, but inhibition of the expression of pTRKB Tyr512 and pAkt was unclear in H460 cells. H810 cells exhibited low or no expression of TRKA/B.

Based on these results, it seems reasonable to conclude that, in KM12 cells, following treatment with AZD7451, phosphorylation of TRKA and cell proliferation were inhibited and these cells are presumably driven through the NTRK1 fusion in vitro. In H460 cells, it was confirmed that the expression of NTRK 2 by western blotting and the phosphorylation of TRKB and cell proliferation were also inhibited by treatment with AZD7451. In H810 cells, we were unable to detect the expression of any of the NTRK1-3 isoforms and cell growth was not inhibited by AZD7451.

In conclusion, we were unable to detect NTRK1 fusions or NTRK1-3 mutations in 268 Japanese NSCLC patients at our institution. One possibility for the discrepancy in these results may be geographical or ethnic differences associated with these patient populations; in addition, the incidence of genetic abnormalities or recurrent polymorphisms in these samples may be significantly lower in Japan compared to Europe or the United States. However, we were able to confirm that a potent and selective inhibitor, such as AZD7451, is able to inhibit the growth of cells with NTRK1 fusions or NTRK2 expression. Therefore, there remains a possibility that NTRK mutations or expression may contribute to the proliferation of lung cancer cells and targeted therapy with a NTRK inhibitor may be of value in the treatment of lung cancer, particularly LCNEC, in the future.

\section{Acknowledgements}

The authors would like to thank Miss Ito Yamamoto for her excellent technical assistance. This study was supported by 
Grants-in-Aid for Scientific Research, Japan Society for the Promotion of Science (nos. 26861125, 25293303 and 24592097).

\section{References}

1. Ginsberg RJ, Kris MK and Armstrong JG: Cancer of the lung. In: Principles and Practice of Oncology. DeVita VT Jr, Hellman S and Rosenberg SA (eds). 4th edition. Lippincott, Philadelphia, PA, pp673-682, 1993.

2. Amano J, Kuwano H and Yokomise H: Thoracic and cardiovascular surgery in Japan during 2011: annual report by the Japanese Association for Thoracic Surgery. Gen Thorac Cardiovasc Surg 61: 578-607, 2013.

3. Postmus PE: Chemotherapy for non-small cell lung cancer: the experience of the Lung Cancer Cooperative Group of the European Organization for Research and Treatment of Cancer. Chest 113 (Suppl 1): 28S-31S, 1998.

4. Shaw AT, Kim DW, Nakagawa K, et al: Crizotinib versus chemotherapy in advanced ALK-positive lung cancer. New Engl J Med 368: 2385-2394, 2013.
5. Mok TS, Wu YL, Thongprasert S, et al: Gefitinib or carboplatin-paclitaxel in pulmonary adenocarcinoma. New Engl J Med 361: 947-957, 2009.

6. Vaishnavi A, Capelletti M, Le AT, et al: Oncogenic and drug-sensitive NTRK1 rearrangements in lung cancer. Nature Med 19: 1469-1472, 2013.

7. Marchetti A, Felicioni L, Pelosi G, et al: Frequent mutations in the neurotrophic tyrosine receptor kinase gene family in large cell neuroendocrine carcinoma of the lung. Human Mut 29: 609-616, 2008

8. Odate $\mathrm{S}$, Nakamura K, Onishi $\mathrm{H}$, et al: TrkB/BDNF signaling pathway is a potential therapeutic target for pulmonary large cell neuroendocrine carcinoma. Lung Cancer 79: 205-214, 2013.

9. Ivanov SV, Panaccione A, Brown B, et al: TrkC signaling is activated in adenoid cystic carcinoma and requires NT-3 to stimulate invasive behavior. Oncogene 32: 3698-3710, 2013.

10. Odate S, Onishi H, Nakayama K, et al: Tropomyosin-related kinase B inhibitor has potential for tumor regression and relapse prevention in pulmonary large cell neuroendocrine carcinoma. Anticancer Res 33: 3699-3703, 2013. 